\title{
Evaluation of Alkali-Pretreated Soybean Straw for Lignocellulosic Bioethanol Production
}

\author{
Seonghun Kim \\ Jeonbuk Branch Institute, Korea Research Institute of Bioscience and Biotechnology, 181 Ipsin-gil, Jeongeup 56212, Republic of Korea \\ Correspondence should be addressed to Seonghun Kim; seonghun@kribb.re.kr
}

Received 17 December 2017; Revised 27 February 2018; Accepted 18 March 2018; Published 3 May 2018

Academic Editor: Xuebing Zhao

Copyright ( 2018 Seonghun Kim. This is an open access article distributed under the Creative Commons Attribution License, which permits unrestricted use, distribution, and reproduction in any medium, provided the original work is properly cited.

\begin{abstract}
Soybean straw is a renewable resource in agricultural residues that can be used for lignocellulosic bioethanol production. To enhance enzymatic digestibility and fermentability, the biomass was prepared with an alkali-thermal pretreatment (sodium hydroxide, $121^{\circ} \mathrm{C}$, $60 \mathrm{~min}$ ). The delignification yield was $34.1 \sim 53 \%$, in proportion to the amount of sodium hydroxide, from 0.5 to $3.0 \mathrm{M}$. The lignin and hemicellulose contents of the pretreated biomass were reduced by the pretreatment process, whereas the proportion of cellulose was increased. Under optimal condition, the pretreated biomass consisted of $74.0 \pm 0.1 \%$ cellulose, $10.3 \pm 0.1 \%$ hemicellulose, and $10.1 \pm 0.6 \%$ lignin. During enzymatic saccharification using Cellic ${ }^{\circledR}$ CTec2 cellulase, $10 \%$ (w/v) of pretreated soybean straw was hydrolyzed completely and converted to $67.3 \pm 2.1 \mathrm{~g} / \mathrm{L}$ glucose and $9.4 \pm 0.5 \mathrm{~g} / \mathrm{L}$ xylose with a $90.9 \%$ yield efficiency. Simultaneous saccharification and fermentation of the pretreated biomass by Saccharomyces cerevisiae W303-1A produced $30.5 \pm 1.2 \mathrm{~g} / \mathrm{L}$ ethanol in $0.5 \mathrm{~L}$ fermented medium containing $10 \%(\mathrm{w} / \mathrm{v})$ pretreated biomass after $72 \mathrm{~h}$. The ethanol productivity was $0.305 \mathrm{~g}$ ethanol/g dry biomass and $0.45 \mathrm{~g}$ ethanol/g glucose after fermentation, with a low concentration of organic acid metabolites. Also, $82 \%$ of fermentable sugar was used by the yeast for ethanol fermentation. These results show that the combination of alkaline pretreatment and biomass hydrolysate is useful for enhancing bioethanol productivity using delignified soybean straw.
\end{abstract}

\section{Introduction}

The soybean (Glycine max L. Merr.) is an annual herbaceous plant in the species of legumes that originated in East Asia (including China, Japan, and Korea), widely grown for its edible bean as an important food resource. During soybean harvesting, the stalks, the husks, and the dry leaves remain as the agricultural residues. In Korean traditional agriculture farming, the dry leaves had been traditionally used as cattle feedstock, but the rest of residues had been consumed as biomass fuel for heating. However, the modern agriculture farm does not use these agricultural residues anymore and abandon the large volumes of the biomass [1].

Nevertheless, soybean straw, the residual part, has the potential to serve an inexpensive feedstock for the production of fermentable sugars, instead of food sources, such as corn, sugar cane, and other food stocks, for the production of bioethanol or other biorefinery products [1, 2]. Among various biomass sources, crop residues such as rice, wheat, barley straw, and corn stover have gained considerable interest and several studies have already been reported based on these feedstocks [3-5]. However, soybean straw, like other lignocellulosic biomaterials, consists of a rigid cellulose structure of strongly cross-linked amorphous hemicellulose and lignin [6-10]. These rigid biomass structures are too chemically complex and too resistant to enzymatic hydrolysis for the production of fermentable sugars. Thus, pretreatment is necessary to change the rigid lignocellulose structures into more enzymatically accessible and digestible forms [2-5].

Soybean straw contains a relatively low level of hemicellulose and lignin per gram biomass, compared with other lignocellulosic biomasses [6-10]. Thus, pretreatment is needed to increase the cellulose content and to decrease the hemicellulose and lignin contents in the biomass. The pretreatment processes should enhance the proportion of cellulose in soybean straw [7-10]. Acidic, alkali, and sequential acidicalkali pretreatments, combined with high temperature or high pressure, have been applied in "conventional" chemical 
treatment processes $[3-5,11-14]$. Acidic pretreatments are known to be effective with lignocellulosic biomasses in reducing the hemicellulose content. Similarly, alkali pretreatments have been reported as simple processes for the delignification of biomass under mild conditions with minimal sugar degradation and without the formation of inhibitory compounds [14]. Thus, chemical pretreatments can reduce hemicellulose and lignin together to enhance the cellulose content of a biomass $[11,13,14]$.

In this study, an alkali-pretreatment was carried out to decrease hemicellulose and lignin for high cellulose content in soybean straw. The biomass was pretreated under different concentration of sodium hydroxide at high temperature, that is, $121^{\circ} \mathrm{C}$. The effectiveness of the alkali-pretreatment of the biomass was determined based on the degree of compositional change and enzymatic hydrolysis of pretreated solids. In addition, to evaluate the alkali-pretreatment process, the simultaneous saccharification and fermentation was also performed using an ethanologenic yeast strain of Saccharomyces cerevisiae and ethanol productivity was determined.

\section{Materials and Methods}

2.1. Materials. Soybean straws were obtained locally from a farm in Jeongeup, Jeonbuk, South Korea, at the end of November 2017. It was washed with water to eliminate soil and other particles and then dried at $105^{\circ} \mathrm{C}$ for $24 \mathrm{~h}$. The straws were chopped into $5-10 \mathrm{~cm}$ lengths for chemical pretreatment. Cellic CTec 2 cellulase was provided by Novozymes Korea (Seoul, Republic of Korea).

2.2. Chemical Pretreatment of Soybean Straws. Dried straws $(20 \% \mathrm{w} / \mathrm{v})$ with no physical treatment were soaked in sodium hydroxide $(\mathrm{NaOH})$ solution in the concentration range of $0 \sim 3 \mathrm{M}$ and heated in an autoclave $\left(121^{\circ} \mathrm{C}, 15 \mathrm{psi}, 60 \mathrm{~min}\right)$. The thermal-alkali-pretreated biomasses were removed from the black alkali solution and then washed with flowing tap water to remove $\mathrm{NaOH}$ from the biomass. This washing step was repeated several times until the washed water showed a pale brown color. The alkali-pretreated straws were dried at $105^{\circ} \mathrm{C}$ for $24 \mathrm{~h}$ to reduce the moisture content and then stored under anhydrous conditions. The composition of the pretreated biomass was analyzed based on the NREL chemical analysis and testing laboratory analytical procedures (LAPs) of the US Department of Energy (DOE). The lignin content of the biomass was analyzed according to the LAPs of the DOE (LAP-003 and LAP-004). The biomass pretreatment with different $\mathrm{NaOH}$ concentration was performed in triplicate.

\subsection{Enzymatic Hydrolysis of the Alkali-Pretreated Soybean} Straw. Enzymatic hydrolysis of the alkali-pretreated straw ( $2 \mathrm{M} \mathrm{NOH}$-treated biomass) was carried out in a tube with a $30 \mathrm{ml}$ reaction volume using Cellic CTec 2 cellulase. 10\% (w/v) pretreated biomass was soaked in phosphate buffer $(\mathrm{pH} 6.0)$ containing the cellulase with 10 50 FPU (filter paper unit) per gram of dry biomass. Enzymatic hydrolysis was performed at $42^{\circ} \mathrm{C}, 200 \mathrm{rpm}$, for $48 \mathrm{~h}$. The biomass hydrolysate was withdrawn at each $12 \mathrm{~h}$ for $72 \mathrm{~h}$. After centrifugation of the enzyme reactants, the hydrolyzed products were analyzed by high-performance liquid chromatography (HPLC) for the amount of monosaccharides generated in the enzymatic hydrolysis. The enzyme solution of Cellic CTec2 cellulase used for the enzymatic hydrolysis basically contains $206 \pm$ $2.3 \mathrm{~g} / \mathrm{L}$ glucose and $193.3 \pm 0.2 \mathrm{~g} / \mathrm{L}$ xylose. The calculation of the amount of monosaccharides (glucose and xylose) hydrolyzed from the pretreated biomass and the enzymatic saccharification yields excludes the amount of these sugars from the solution of the cellulase.

2.4. Ethanol Production Strain, Growth Conditions, and Fermentation. Saccharomyces cerevisiae W303-1A was used as an ethanol production strain [12]. The ethanologenic strain was cultivated in YPD broth (2\% Bacto peptone, $1 \%$ Bacto yeast extract, and $2 \%$ glucose) at $30^{\circ} \mathrm{C}$ at $200 \mathrm{rpm}$ for $24 \mathrm{~h}$. To prepare a seed culture for ethanol fermentation, $1 \%(\mathrm{v} / \mathrm{v})$ seed culture was inoculated in a medium containing enzymatic hydrolysis solution, in which $10 \%(\mathrm{w} / \mathrm{v})$ pretreated soybean straw was hydrolyzed by the cellulase for $48 \mathrm{~h}$ as carbon sources, 2\% Bacto peptone, and 1\% Bacto yeast extract as nutrient sources. Then the seed culture was further cultivated at $30^{\circ} \mathrm{C}$ at $200 \mathrm{rpm}$ for $24 \mathrm{~h} .5 \%(\mathrm{v} / \mathrm{v})$ preculture of the yeast strain was inoculated into a 1-L fermentor FMT ST-S (Fermentec, Cheongju, South Korea) with a $0.5 \mathrm{~L}$ working volume containing $10 \%(\mathrm{w} / \mathrm{v})$ alkali-pretreated soybean straw, $2 \%$ Bacto peptone, and $1 \%$ Bacto yeast extract and then the fermentor was operated at $30^{\circ} \mathrm{C}$ with agitation at $300 \mathrm{rpm}$. The ethanol fermentation was performed in triplicate.

2.5. Analytical Procedures. Cell growth was monitored by measuring the optical density at $600 \mathrm{~nm}$ (OD600 nm) using a spectrophotometer. Total reducing sugars in the enzymatic saccharification reaction were measured using the 3,5dinitrosalicylic acid method. The amounts of the released sugars in the enzymatic saccharification and the metabolites in fermentation were determined with a high-performance liquid chromatography (HPLC) system (Agilent Technologies, Santa Clara, CA, USA), equipped with a refractive index detector, an autosampler, and an Aminex HPX-87P column $(7.8 \times 300 \mathrm{~mm}$; BioRad, Hercules, CA, USA $)$ for monosaccharide analysis or a Rezex ROA-Organic Acid $\mathrm{H}^{+}$ column $(7.8 \times 300 \mathrm{~mm}$; Phenomenex, Torrance, CA, USA) for organic acid analysis. All samples were clarified by filtration with a $0.20-\mu \mathrm{m}$ filter (Acrodisc LC PVDF Minispike; Pall Life Sciences, Ann Arbor, MI, USA) and then injected into the analytical HPLC column. The column temperature was kept at $65^{\circ} \mathrm{C}$. The mobile phase was distilled water for monosaccharides and $2.5 \mathrm{mM}$ sulfuric acid for organic acids, with a flow rate $0.5 \mathrm{~mL} / \mathrm{min}$ under isocratic conditions. Under these conditions, cellobiose, glucose, xylose, ethanol, glycerol, and xylitol were detected at the retention times 9.93, 12.08, 13.14, $16.36,18.96$, and $34.33 \mathrm{~min}$, respectively, in monosaccharide analyses. Succinic acid, lactic acid, acetic acid, and ethanol were detected at the retention times 13.14, 16.36, 18.96, and $34.33 \mathrm{~min}$, respectively, in organic acid analyses. All analyses were performed in triplicate 
TABLE 1: The chemical composition of soybean straws.

\begin{tabular}{|c|c|c|c|c|c|c|}
\hline \multirow[b]{2}{*}{ Sample } & \multicolumn{6}{|c|}{ Compositions (\%) per $100 \mathrm{~g}$ soybean straw* } \\
\hline & 1 & 2 & 3 & 4 & 5 & 6 \\
\hline Cellulose & $44-83$ & 25.0 & 34.1 & 39.8 & 35.3 & 44.2 \\
\hline Hemicellulose & - & 11.9 & 16.1 & 22.6 & 16.9 & 5.9 \\
\hline $\operatorname{Lignin}^{(2)}$ & $5-14$ & 17.6 & $21.6^{(1)}$ & 12.8 & $21.7^{(1)}$ & $19.2^{(1)}$ \\
\hline Ash & $2-5$ & - & 5.2 & - & 10.6 & - \\
\hline Reference & {$[6]$} & [7] & [8] & {$[9]$} & {$[10]$} & This study \\
\hline
\end{tabular}

\section{Results and Discussion}

3.1. Alkali Treatment of Soybean Straw and Its Composition. Prior to alkali-pretreatment, cellulose, hemicellulose, and lignin contents in the soy straw raw material were determined by using the methods of the US DOE. Dried soybean straw $(100 \mathrm{~g})$ consisted of $44.2 \%$ cellulose, $5.9 \%$ hemicellulose, and $19.2 \%$ lignin (Table 1). Compared to these contents in several different biomasses summarized in Table 1 [6-10], the soybean straw used in this study contained a relatively low amount of hemicellulose and a high content of lignin. It needs to reduce lignin content to enhance the enzymatic accessibility and digestibility to produce a fermentable sugar glucose and to enhance ethanol production yield using an ethanologenic strain [5].

To enhance a high-cellulose-content biomass, alkalipretreatment was applied to reduce lignin in the soybean straw (Figure 1). After the biomass had dried completely, the chemical composition change in alkali-pretreated soybean straws was analyzed (Table 2). The concentration of sodium hydroxide affected the solubility of the biomass and the loss of cellulose, hemicellulose, and lignin contents. Alkalithermal treatment within sodium hydroxide extracted up to $52 \sim 64.5 \%$ of the biomass into the soluble fraction, even though hot water without any alkali compound extracted $29.1 \%$ of the biomass. With $3 \mathrm{M}$ sodium hydroxide pretreatment, the insoluble residue fraction contained $72.9 \%$ cellulose, 9.1\% hemicellulose, and 9.0\% lignin per $100 \mathrm{~g}$ dry biomass. After alkali treatment, 9.0 12.6\% lignin and 9.1 17.9\% hemicellulose remained in the residual biomass. However, the pretreated biomass did not lose much cellulose content, from $66.4 \mathrm{~g}$ to $72.9 \mathrm{~g}$ per $100 \mathrm{~g}$ of dry soybean straw, with an increase in the sodium hydroxide concentration. The delignification yield with the alkali-thermal pretreatment was $34.1 \sim 53.0 \%$, in proportion to the amount of sodium hydroxide, from 0.5 to $3.0 \mathrm{M}$. However, $>47 \%$ of the lignin could not be removed from the biomass under the alkalithermal pretreatment conditions, even with a high concentration of sodium hydroxide. Nevertheless, the alkali-thermal treatment of the biomass showed that sodium hydroxide reduces hemicellulose and lignin effectively and increases the cellulose content per gram biomass.

3.2. Enzymatic Hydrolysis of Soybean Straw. To assess the enzymatic hydrolysis of alkali-thermal-pretreated soybean

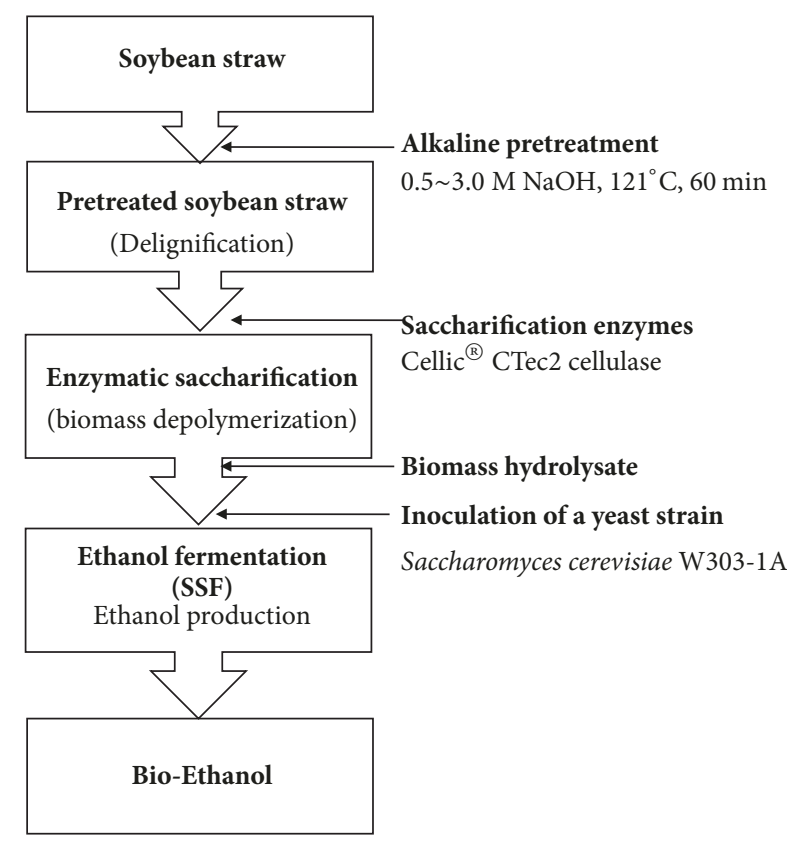

FIGURE 1: Overall procedure for the simultaneous saccharification and fermentation of the alkali-treated soybean straw for ethanol production.

straw with $2 \mathrm{M}$ sodium hydroxide, the pretreated biomass $(10 \%(\mathrm{w} / \mathrm{v}))$ was digested with different enzyme dose of Cellic CTec2 cellulase, from 10 to 50 FPU per gram of dry biomass in phosphate buffer $(\mathrm{pH} \mathrm{6.0)}$ for $72 \mathrm{~h}$. The $\mathrm{pH}$ in the reaction media for the enzymatic digestion could affect the growth, metabolism, and transport in an ethanologenic strain for further ethanol fermentation [15]. Thus $\mathrm{pH}$ was fixed at 6.0 for the enzymatic biomass saccharification. The amount of glucose produced by enzymatic hydrolysis increased with increasing amounts of cellulase per unit the alkali-pretreated soybean straw (Figure 2(a)). At $72 \mathrm{~h}$, the cellulose generated 40.1 67.3 g/L glucose and 5.6 9.4 g/L xylose. The enzymatic digestibility at the enzyme loading ratio of $50 \mathrm{FPU}$ cellulase per gram of dry biomass reached $54.2 \pm 2.8 \% \sim 90.9 \pm 2.8 \%$ (Figure 2(b)). On the other hand, the nontreated biomass was hydrolyzed with less than 33\% (data now shown). Compared with untreated soybean straw, the alkali-thermal-pretreated biomass contained higher amounts of glucose and lower amounts of xylose. Alkali-pretreatment with approximate 


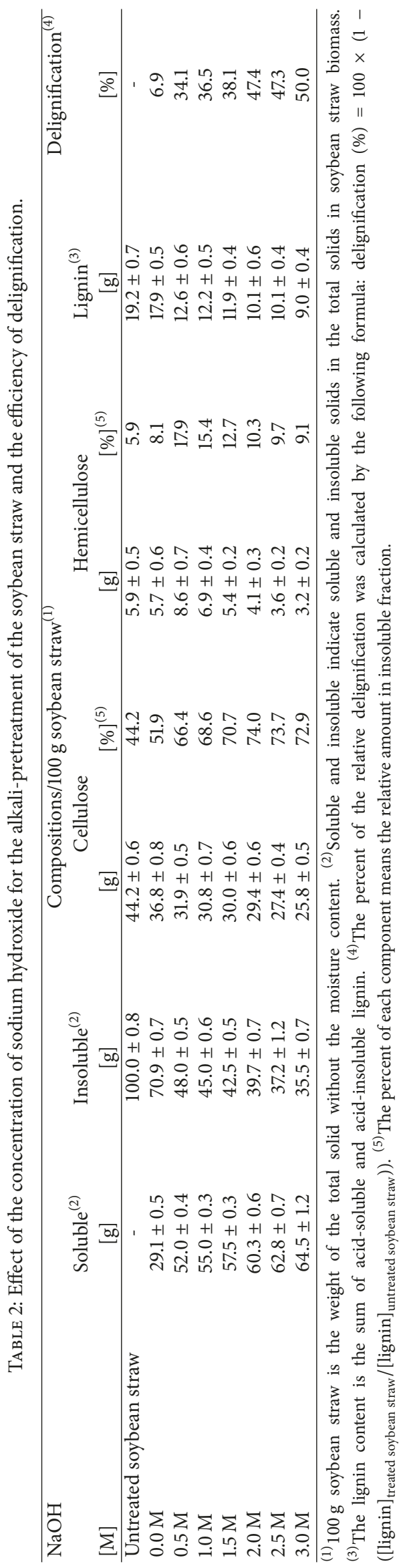




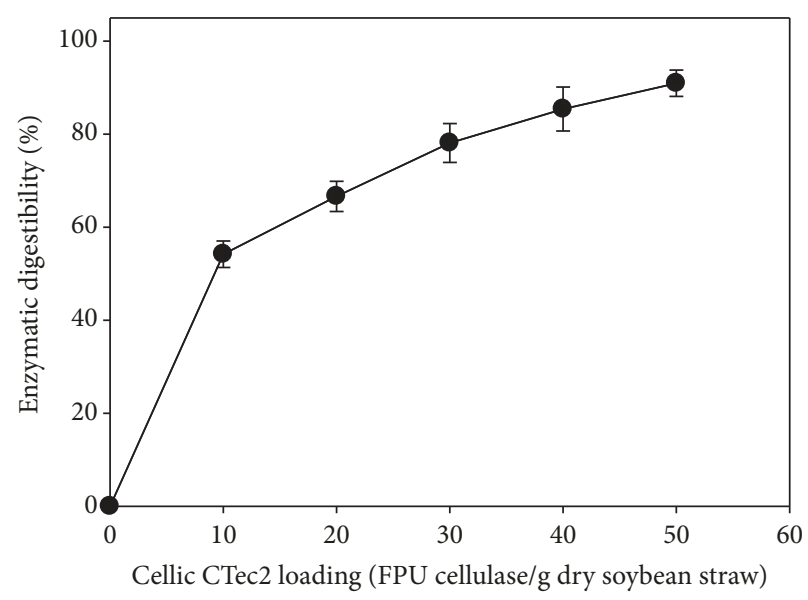

(a)

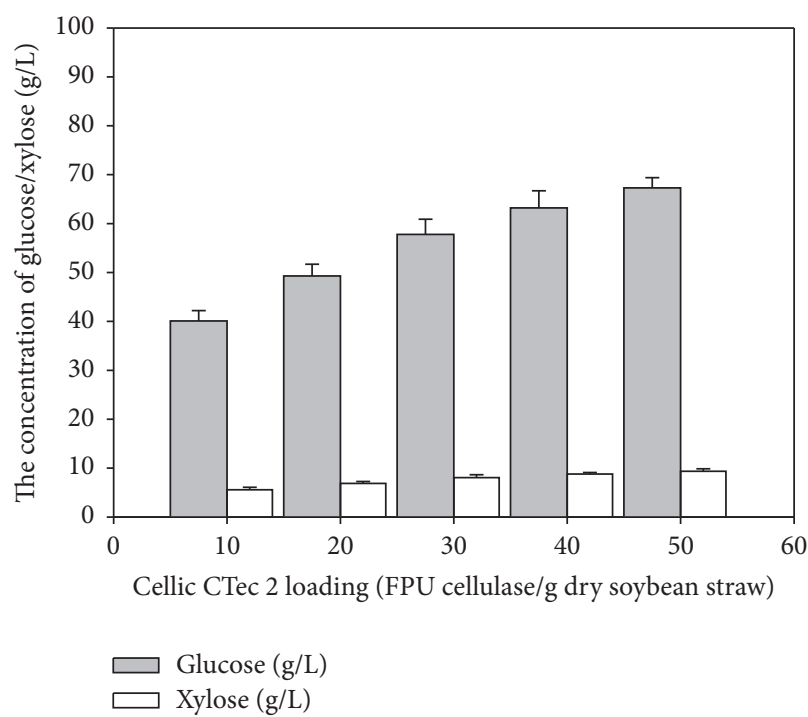

(b)

Figure 2: Enzymatic saccharification of the alkali-treated soybean straw with 50 FPU of Cellic CTec2 cellulase loading per gram of the dry biomass. The enzymatic digestibility was calculated by the relative amount of glucose released from the content of glucan in the pretreated biomasses after enzyme reaction.

$47.4 \%$ delignification efficiency would affect the cellulase digestibility, resulting in a hydrolysis efficiency up to $90 \%$. The chemical pretreatment also reduced the strong interactions in cellulose/hemicellulose/lignin complexes in the biomass structure, enhancing the enzyme reaction efficiency. An increase in the volume of enzymes used significantly affects the amount of fermentable sugar generation. At the final reaction time point, the amount of glucose increased up to 1.7 times depending on 5 enzyme loading ratios of Cellic CTec2 cellulase per biomass. Additionally, sugar production increased time-dependently, whereas enzymatic hydrolysis rates decreased exponentially due to product inhibition (data not shown). The generated glucose, xylose, and incompletely digested cellobiose may inhibit the hydrolysis reactions of the cellulase. These enzymatic saccharifications showed that alkali-pretreated soybean straw could be powerful to prepare a fermentable sugar glucose for bioethanol production.

\subsection{Separate Hydrolysis and Fermentation of Alkali-Pretreated} Soybean Straw. To evaluate the fermentability of the sugars generated from the alkali-pretreated soybean straw, ethanol production was performed using Saccharomyces cerevisiae W303-1A [12]. Batch cultivation of the yeast strain was performed in a $50-\mathrm{mL}$ culture volume in a $250-\mathrm{mL}$ Erlenmeyer flask with $5 \mathrm{~g}$ of alkali-pretreated soybean straw, supplemented with a $50 \mathrm{FPU}$ Cellic CTec2 cellulase. Before cell inoculation, prehydrolysis was performed at $42^{\circ} \mathrm{C}$ for $12 \mathrm{~h}$. Then, $5 \%(\mathrm{v} / \mathrm{v})$ yeast inoculum was added and cultured further $\left(30^{\circ} \mathrm{C}, 180 \mathrm{rpm}, 36 \mathrm{~h}\right)$ for ethanol fermentation. The prehydrolysis step generated $\sim 12.1 \pm 0.7 \mathrm{~g} / \mathrm{L}$ glucose in the flask for yeast cell growth without the lag phase. At $36 \mathrm{~h}$, the maximum ethanol concentration of $13.1 \pm 0.7 \mathrm{~g} / \mathrm{L}$ was

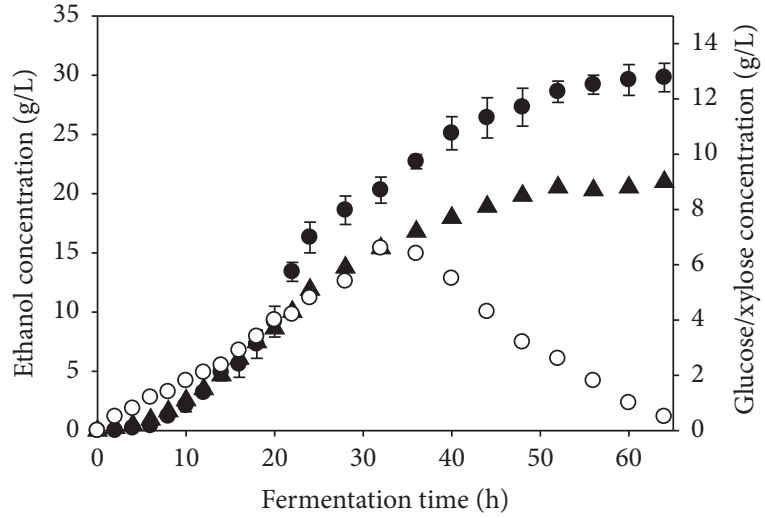

FIGURE 3: Batch simultaneous saccharification and fermentation of the alkali-pretreated soybean straw by Saccharomyces cerevisiae W303-1A in a 1-L jar fermenter. Profiles of ethanol (filled circle), glucose (open circle), and xylose (filled triangle) in the fermenter were analyzed by HPLC.

observed in the culture broth. However, low ethanol production yield was observed in the flask culture. The enzymatic saccharification was not efficient in the flask culture.

\subsection{Batch Simultaneous Saccharification and Fermentation} (SSF) for Ethanol Production. To increase ethanol production, ethanol fermentation was performed in a 1-L jar fermentor. For batch SSF, $10 \%(\mathrm{w} / \mathrm{v})$ pretreated biomass was enzymatically saccharified with 50 FPU of Cellic CTec2 cellulase per gram of dry biomass. The ethanol production proceeded gradually for $72 \mathrm{~h}$ (Figure 3). The amount of glucose was gradually increased by enzymatic hydrolysis. Although the ethanologenic yeast consumed glucose for cell growth, the 
concentration of glucose generated exceeded the amount consumed. The yeast completely consumed all glucose enzymatically hydrolyzed from the biomass in the medium over $60 \mathrm{~h}$. Nevertheless, the amount of xylose increased gradually to $9.1 \pm 0.5 \mathrm{~g} / \mathrm{L}$, because the wild type $S$. cerevisiae W303$1 \mathrm{~A}$ can not utilize the sugar. In a batch SSF, the maximum ethanol concentration was $30.5 \pm 1.2 \mathrm{~g} / \mathrm{L}$ at $72 \mathrm{~h}$, giving an ethanol production yield of $0.305 \mathrm{~g}$ ethanol/g dry soybean straw and $0.45 \mathrm{~g}$ ethanol/g glucose at $72 \mathrm{~h}$. Finally, $82 \%$ of the fermentable sugar hydrolyzed from the pretreated biomass was used by the yeast for ethanol production. In addition, the decrease of ethanol concentration was not observed until glucose was completely depleted in the fermentation $(72 \mathrm{~h})$.

In ethanol production of the alkali-pretreated soybean straw, the baker's S. cerevisiae W303-1A generated minor metabolites, including glycerol and organic acids such as acetic acid, lactic acid, and succinic acid (data not shown). These metabolites are less than $1.5 \pm 0.4 \mathrm{~g} / \mathrm{L}$. These minor byproducts generated by the yeast were at lower concentrations than ethanol. Although these metabolites remained and were accumulated during the ethanol production, it did not affect the fermentation efficiency or the yeast cell growth.

In this study, an alkali-pretreatment was applied to an agricultural residue soybean straw as a potential bioresource to produce fermentable sugars by enzymatic saccharification. The soybean straw used in this pretreatment contained relatively high cellulose and lignin contents rather than hemicellulose. To reduce lignin and to enhance cellulose content, sodium hydroxide among chemical pretreatments was chosen for delignification of soybean straw. The maximum cellulose content of $74 \%$ was achieved with $2 \mathrm{M} \mathrm{NaOH}$ at $121^{\circ} \mathrm{C}$ after $60 \mathrm{~min}$ and, at this condition, delignification of $47.4 \%$ and hemicellulose content of $10.3 \%$ (Table 2 ).

In fact, the advantage of sodium hydroxide pretreatment can break down effectively the internal structure of lignocellulose component through degrading the ester and glycosidic chains. In addition, the alkali can alter the structure of lignin, causing cellulose swelling and partial decrystallization of cellulose [16]. Moreover the safer handling and the lower toxicity in the pretreatment, including lower operating temperatures and faster process times, make it an attractive alternative to ammonia pretreatment [17]. Relatively lower thermal-pretreatment condition also reduce sugar degradation products such as furfural, hydroxymethylfurfural, and organic acids, considering potential inhibitors for enzymatic saccharification as well as ethanol fermentation [18]. In enzymatic digestion and the fermentation process, these inhibitors were not detected in the biomass lysate. Moreover, the soybean straw pretreated with $2 \mathrm{M} \mathrm{NaOH}$ was effectively hydrolyzed with a hydrolysis efficiency up to $90 \%$ for production of the fermentable sugars (Figure 2(a)). Additionally, the simultaneous saccharification and fermentation converted the sugar of the biomass hydrolysate to $30.5 \mathrm{~g} / \mathrm{L}$ of ethanol with ethanol conversion of more than $82 \%$ (Figure 3 ).

Although the concentration of sodium hydroxide for pretreatment of soybean straw was relatively higher than other alkali chemical compounds, sodium hydroxide solution could be recovered and reused several times for biomass pretreatment [19]. In addition, compared with other chemical pretreatments using aqueous ammonia, organosolv, and ionic liquids, the sodium hydroxide-treatment could be a simple and convenient process, because it does not require any special equipment or control systems for the pretreatment [19].

The utilization of soybean straw could offer several advantages for the sustainable development based on the biomass utilization. Sugar crops or alternative lignocellulosic biomass plants consumed nutrients in the soil leading to decreased nutrients levels. On the other hand, a legume plant soybean with symbiotic bacteria Rhizobia in the nodules of its root systems can fix nitrogen into ammonia and ammonium leading to nitrogen $(\mathrm{N})$ enrichment in the soil, including $\mathrm{CO}_{2}$ fixation by photosynthesis [20]. Considering the sustainability, the residual biomass from the soybean is a potential resource for production of fermentable sugar.

\section{Conclusions}

The alkali-pretreatment of soybean straw with sodium hydroxide effectively removed lignin and hemicellulose and enhanced enzyme digestibility. The alkali-pretreated biomass showed $\sim 53 \%$ delignification efficiency. In the pretreated biomass, over $90 \%$ of the cellulose was hydrolyzed by Cellic CTec2 cellulase and was converted to fermentable sugars during enzymatic saccharification. In a flask-scale culture of separate hydrolysis and fermentation supplemented with the pretreated biomass, S. cerevisiae produced $13.1 \mathrm{~g} / \mathrm{L}$ ethanol with $0.13 \mathrm{~g}$ ethanol/g biomass. In a batch of simultaneous hydrolysis and fermentation, the pretreated soybean straw was converted to $30.5 \mathrm{~g} / \mathrm{L}$ of ethanol with the product yield of $0.305 \mathrm{~g}$ ethanol/g dry soybean straw and $0.45 \mathrm{~g}$ ethanol $/ \mathrm{g}$ glucose. In the fermentation, $82 \%$ fermentable sugar was converted to ethanol within a low concentration of organic acid byproducts. These results clearly show that alkalipretreatment for an agricultural byproduct soybean straw efficiently reduces lignin and hemicellulose components and increases enzymatic digestibility and ethanol productivity.

\section{Conflicts of Interest}

The author declares no conflicts of interest regarding the publication of this article.

\section{Acknowledgments}

This work was partially supported by Research Exchange Program under Memorandum of Understanding between the NRF-DAAD (NRF-2013K2A5A5079255) from Korea National Research Foundation (NRF), the New \& Renewable Energy Technology Development Program of the Korea Institute of Energy Technology Evaluation and Planning (KETEP) granted financial resource from the Ministry of Trade, Industry \& Energy, Republic of Korea (no. 20153030101580), and KRIBB Research Initiative Program grant. 


\section{References}

[1] M. Caicedo, J. Barros, and B. Ordás, "Redefining agricultural residues as bioenergy feedstocks," Materials, vol. 9, no. 8, article no. E635, 2016.

[2] S. Brethauer and M. H. Studer, "Biochemical conversion processes of lignocellulosic biomass to fuels and chemicals - A review," Chimia, vol. 69, no. 10, pp. 572-581, 2015.

[3] L. Capolupo and V. Faraco, "Green methods of lignocellulose pretreatment for biorefinery development," Applied Microbiology and Biotechnology, vol. 100, no. 22, pp. 9451-9467, 2016.

[4] L. P. Devendra, M. Kiran Kumar, and A. Pandey, "Evaluation of hydrotropic pretreatment on lignocellulosic biomass," Bioresource Technology, vol. 213, pp. 350-358, 2016.

[5] M. H. L. Silveira, A. R. C. Morais, A. M. Da Costa Lopes et al., "Current Pretreatment Technologies for the Development of Cellulosic Ethanol and Biorefineries," ChemSusChem, vol. 8, no. 20, pp. 3366-3390, 2015.

[6] N. Reddy and Y. Yang, "Natural cellulose fibers from soybean straw," Bioresource Technology, vol. 100, no. 14, pp. 3593-3598, 2009.

[7] Z. Xu, Q. Wang, Z. Jiang, X.-X. Yang, and Y. Ji, "Enzymatic hydrolysis of pretreated soybean straw," Biomass \& Bioenergy, vol. 31, no. 2-3, pp. 162-167, 2007.

[8] C. Wan, Y. Zhou, and Y. Li, "Liquid hot water and alkaline pretreatment of soybean straw for improving cellulose digestibility," Bioresource Technology, vol. 102, no. 10, pp. 62546259, 2011.

[9] M. Martelli-Tosi, O. B. G. Assis, N. C. Silva, B. S. Esposto, M. A. Martins, and D. R. Tapia-Blácido, "Chemical treatment and characterization of soybean straw and soybean protein isolate/straw composite films," Carbohydrate Polymers, vol. 157, pp. 512-520, 2017.

[10] E. Cabrera, M. J. Muñoz, R. Martín, I. Caro, C. Curbelo, and A. B. Díaz, "Comparison of industrially viable pretreatments to enhance soybean straw biodegradability," Bioresource Technology, vol. 194, pp. 1-6, 2015.

[11] S. Kim, J. M. Park, J.-W. Seo, and C. H. Kim, "Sequential acid-/alkali-pretreatment of empty palm fruit bunch fiber," Bioresource Technology, vol. 109, pp. 229-233, 2012.

[12] S. Kim and C. H. Kim, "Bioethanol production using the sequential acid/alkali-pretreated empty palm fruit bunch fiber," Journal of Renewable Energy, vol. 54, pp. 150-155, 2013.

[13] S. Kim, J. M. Park, and C. H. Kim, "Ethanol production using whole plant biomass of Jerusalem artichoke by Kluyveromycs marxianus CBS1555," Applied Biochemistry and Biotechnology, vol. 169, no. 5, pp. 1531-1545, 2013.

[14] S. Kim and C. H. Kim, "Evaluation of whole Jerusalem artichoke (Helianthustuberosus L.) for consolidated bioprocessing ethanol production," Journal of Renewable Energy, vol. 65, pp. 83-91, 2014.

[15] A. Peña, N. S. I. Sánchez, H. Álvarez, M. Calahorra, and J. Ramírez, "Effects of high medium $\mathrm{pH}$ on growth, metabolism and transport in Saccharomyces cerevisiae," FEMS Yeast Research, vol. 15, no. 2, pp. 1-13, 2015.

[16] Y. Jiang, X. Zeng, R. Luque et al., "Cooking with active oxygen and solid alkali: a promising alternative approach for lignocellulosic biorefineries," ChemSusChem, vol. 10, no. 20, pp. 3982-3993, 2017.

[17] A. D. Moreno, D. Ibarra, P. Alvira, E. Tomás-Pejó, and M. Ballesteros, "A review of biological delignification and detoxification methods for lignocellulosic bioethanol production,"
Critical Reviews in Biotechnology, vol. 35, no. 3, pp. 342-354, 2015.

[18] H. Rasmussen, H. R. Sørensen, and A. S. Meyer, "Formation of degradation compounds from lignocellulosic biomass in the biorefinery: Sugar reaction mechanisms," Carbohydrate Research, vol. 385, pp. 45-57, 2014.

[19] M. Vochozka, V. Stehel, and A. Maroušková, “Daphnia magna demonstrated sufficient sensitivity in techno-economic optimization of lignocellulose bioethanol production," 3 Biotech, vol. 7, no. 3, article no. 162, 2017.

[20] P. M. Gresshoff, S. Hayashi, B. Biswas et al., "The value of biodiversity in legume symbiotic nitrogen fixation and nodulation for biofuel and food production," Journal of Plant Physiology, vol. 172, pp. 128-136, 2015. 


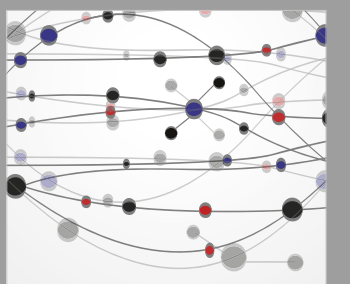

The Scientific World Journal
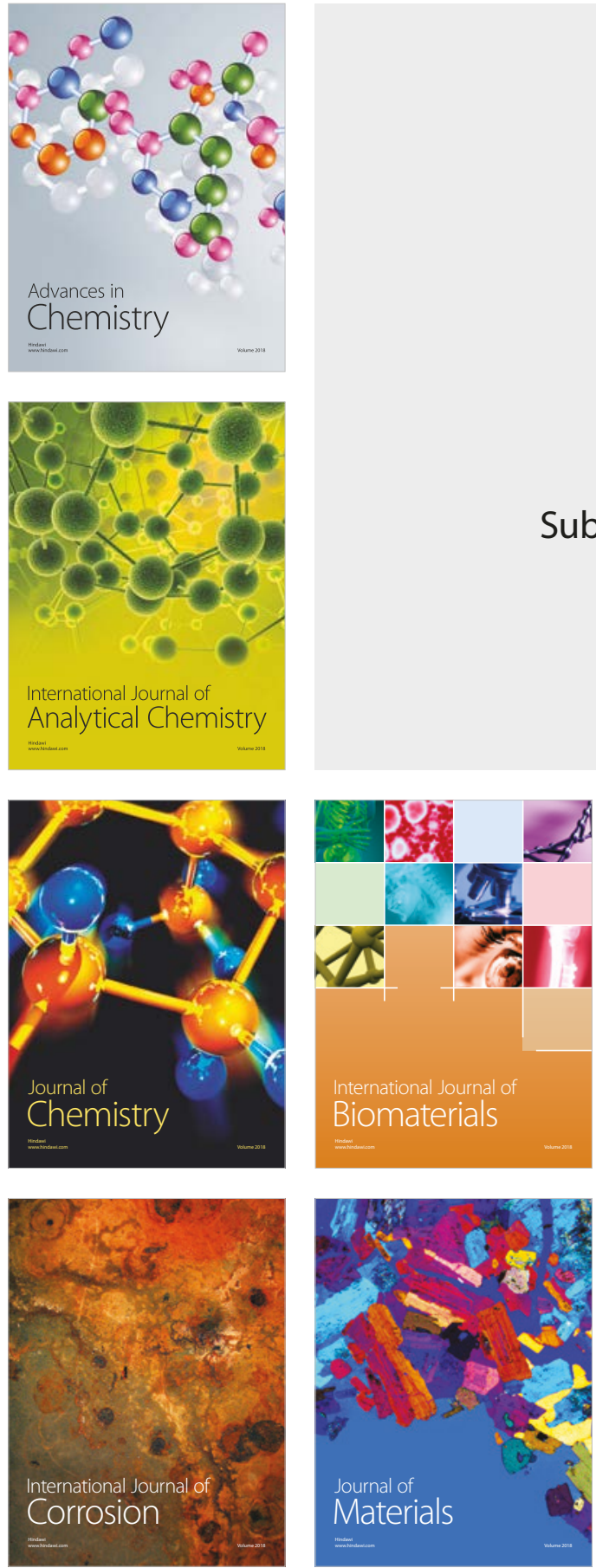

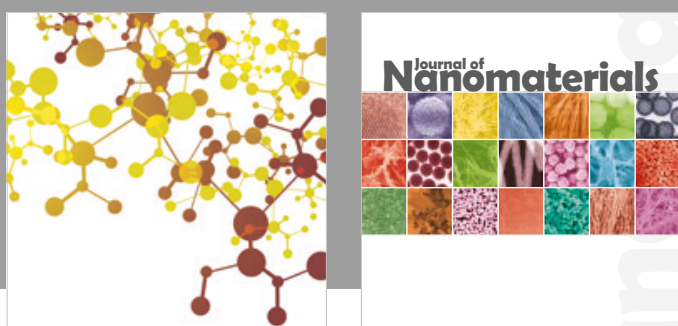

Journal of

Applied Chemistry

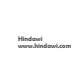

Submit your manuscripts at

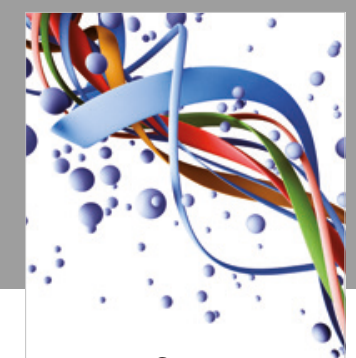

Scientifica
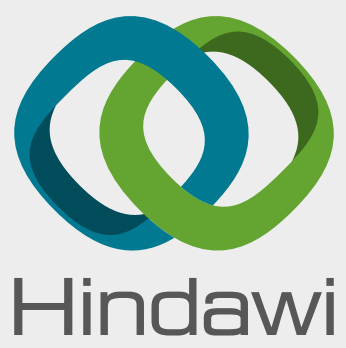

www.hindawi.com
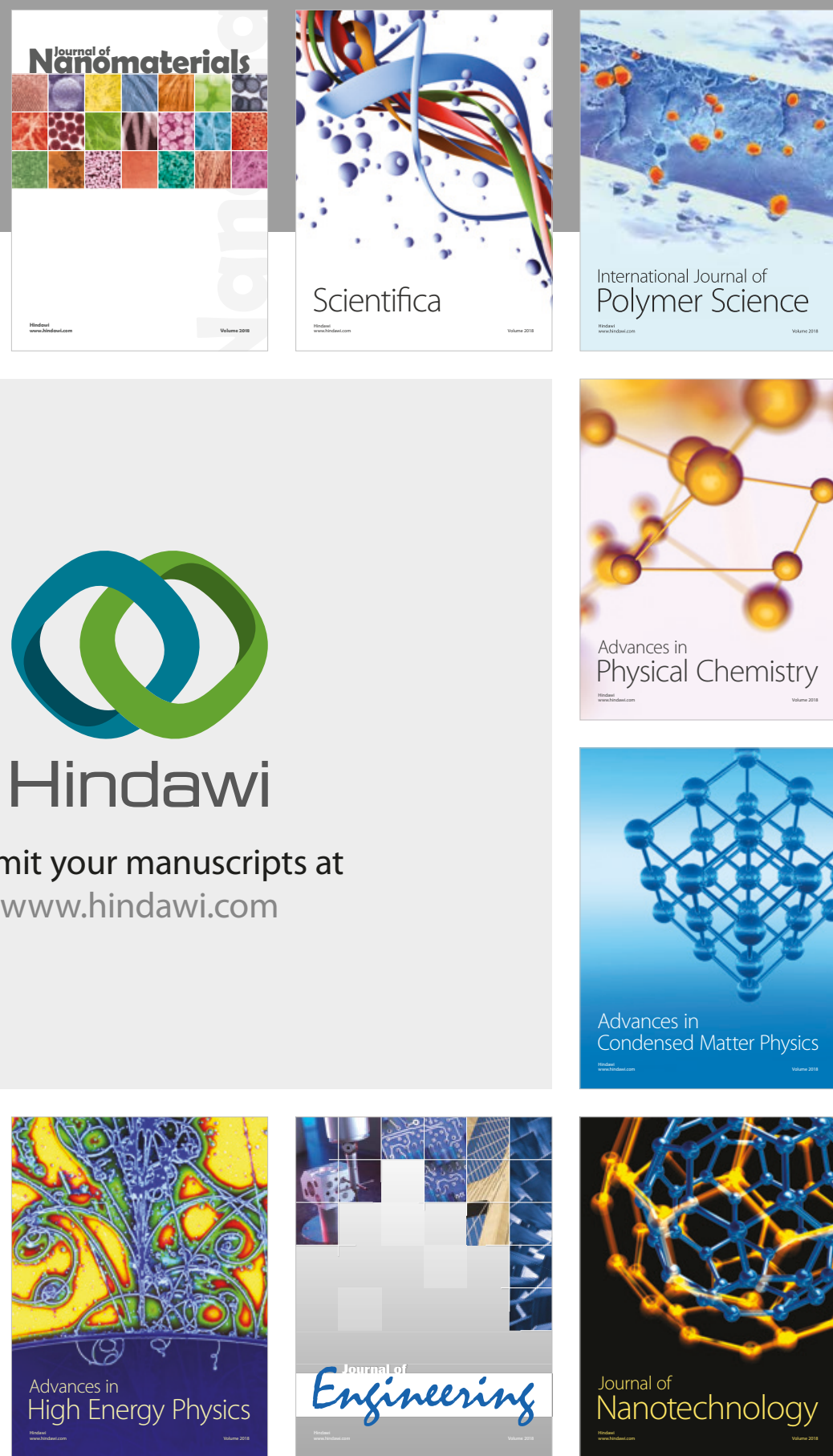

Polymer Science

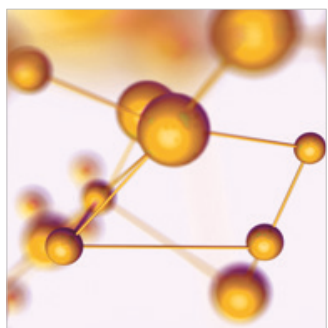

Advances in

Physical Chemistry
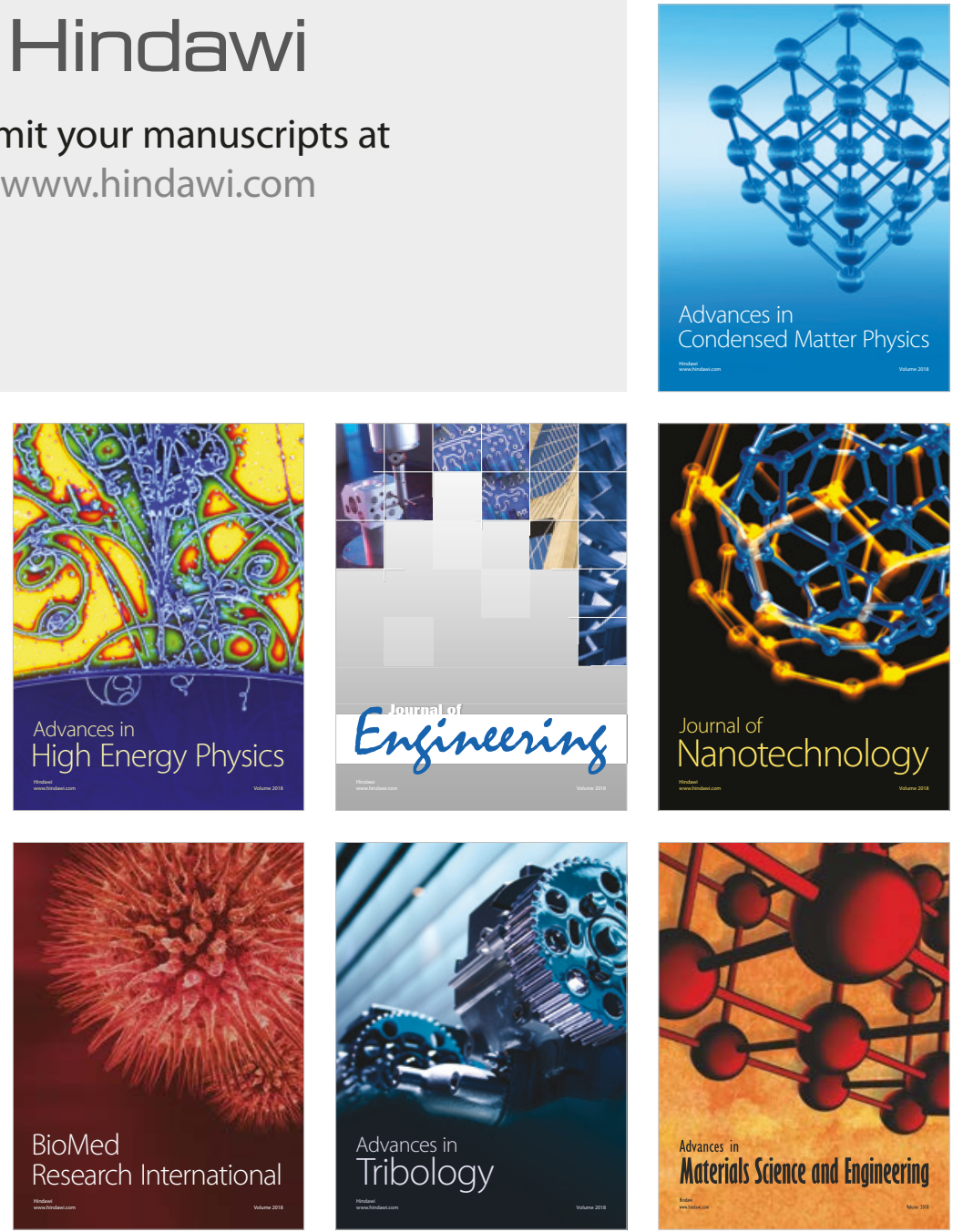